\title{
A Fermi Energy-Incorporated Framework for Dealing with the Temperature- and Magnetic Field-Dependent Critical Current Densities of Superconductors and Its Application to Bi-2212
}

\author{
Gulshan Prakash Malikㅁ, Vijaya Shankar Varma² \\ ${ }^{1}$ B-208, Sushant Lok 1, Gurgaon, Haryana, India \\ ${ }^{2} 180$ Mall Apartments, Delhi, India \\ Email: gulshanpmalik@yahoo.com,varma2@gmail.com
}

How to cite this paper: Malik, G.P. and Varma, V.S. (2020) A Fermi Energy-Incorporated Framework for Dealing with the Temperature- and Magnetic Field-Dependent Critical Current Densities of Superconductors and Its Application to Bi-2212. World Journal of Condensed Matter Physics, 10, 53-70.

https://doi.org/10.4236/wjcmp.2020.102004

Received: February 6, 2020

Accepted: March 15, 2020

Published: March 18, 2020

Copyright $\odot 2020$ by author(s) and Scientific Research Publishing Inc. This work is licensed under the Creative Commons Attribution International License (CC BY 4.0).

http://creativecommons.org/licenses/by/4.0/

\begin{abstract}
It is well known that the critical current density of a superconductor depends on its size, shape, nature of doping and the manner of preparation. It is suggested here that the collective effect of such differences for different samples of the same superconductor is to endow them with different values of the Fermi energy - a single property to which may be attributed the observed variation in their critical current densities. The study reported here extends our earlier work concerned with the generalized BCS equations [Malik, G.P. (2010) Physica B, 405, 3475-3481; Malik, G.P. (2013) WJCMP, 3, 103-110]. We develop here for the first time a framework of microscopic equations that incorporates all of the following parameters of a superconductor: temperature, momentum of Cooper pairs, Fermi energy, applied magnetic field and critical current density. As an application of this framework, we address the different values of critical current densities of Bi-2212 for non-zero values of temperature and applied magnetic field that have been reported in the literature.
\end{abstract}

\section{Keywords}

Generalized BCS Equations, Fermi Energy, Critical Current Density,

Non-Zero Temperatures and Applied Fields, Bi-2212

\section{Introduction}

The critical current density $\left(j_{c}\right)$ of a superconductor (SC) is an important parameter because the greater its value, the greater is the practical use to which it can 
be put. Most of the plethora of formulae available in the literature for calculating this parameter may be mainly categorized as following from the framework of the Londons' equations or the Ginsburg-Landau (GL) equations. The salient features of such approaches are that they are based on diverse criteria such as the type of SC being dealt with (type I or II) and its geometry [1] [2] [3] [4] [5]. Another limitation of both the Londons' and the GL theories is that they work for the $j_{c}$ of an SC only when its temperature $\mathrm{T}$ is close to its critical temperature $T_{c}$ In contrast with these phenomenological approaches, there is also available in the literature a smaller body of work dealing with the $j_{c}$ of an SC on the basis of the microscopic theory of superconductivity. Notable among these is the often-used approach of Kupriyanov and Lukichev [6] based on a simplification of the Eilenberger theory, which in turn is derived from the original Gor'kov theory under the assumption that $\rho_{F} l \gg 1$, where $\rho_{F}$ is the electrical resistivity of electrons at the Fermi surface and $l$ their mean free path.

An overview of the study reported herein is as follows. Guided by a substantial body of recent work, e.g., [6]-[12], which suggests that low values of the Fermi energy $\left(E_{F}\right)$ play a pivotal role in determining properties of high- $T_{c} S C s$, we have been following a course where $E_{F}$ is directly incorporated into the generalized BCS equations for the gaps $(\Delta s)$ and the $T_{c} s$ of both elemental and composite SCs [13] [14]. This is a departure from the usual practice since these parameters are conventionally calculated via equations independent of $E_{F}$ because of the assumption that $E_{F} / k \theta \gg 1$, where $k$ is the Boltzmann constant and $\theta$ the Debye temperature of the SC. As a supplement to this framework, we reported in [15] and [16] the results of an exercise that includes an $E_{F}$-incorporated equation for $\mathrm{j}_{\mathcal{c}}$, leading to a unified framework for dealing with the $\Delta_{\mathcal{s}}, T_{\mathcal{c}} s$ and $j_{\mathcal{c}} s$ of both elemental and composite SCs at $T=0$ and $H=0$, where $\mathrm{H}$ is the applied external magnetic field. Since the $j_{c}$-values of an SC are generally reported for non-zero values of both $T$ and $H$, we present here a framework to deal with such a situation. In essence, the present work is concerned with a generalization of: 1) the work reported in [17] to include $E_{F}$ in the pairing equations that already incorporate $T$ and $H$ and 2) the work reported in [18] to include $H$ in the equations that already incorporate $T, E_{F}$ and the momentum $\boldsymbol{P}$ of the pairs. We believe that presented herein is the first attempt that brings the $T$-and $H$-dependent $j_{c}$ of an SC under the purview of an $E_{F}$-incorporated microscopic theory of superconductivity.

The paper is organized as follows. In Section 2, we show how, without making the usual approximation $E_{F} \gg k \theta$, the dynamical equation for a Cooper pair interacting via the model BCS interaction can be generalized to include $T$ and $H$ via the Matsubara prescription and the Landau quantization scheme, respectively. We thus obtain the pairing equation incorporating $E_{F}, T$ and $H$ corresponding to the 1-phonon exchange mechanism (1PEM) and $\boldsymbol{P}=0$, which is appropriate for dealing with the situation when $j=0$. In order to deal with the situation when $j \neq 0$, we need to do away with the $\boldsymbol{P}=0$ constraint of this section. 
This is done in Section 3, assuming that the dimensionless interaction parameter remains unchanged as we move out of the center-of-mass frame to the lab frame. Based on the equations thus obtained, in Section 4 are derived expressions for the density of the superconducting electrons $\mathrm{n}_{s}$, their critical velocity $v_{\mathcal{c}}, j_{c}$ and $s \equiv m^{*} / m_{e}$, where $m^{*}$ is the effective mass of an electron and $m_{e}$ the free electron mass. Application of these equations to deal with the empirical data of $\mathrm{Bi}_{2} \mathrm{Sr}_{2} \mathrm{CaCu}_{2} \mathrm{O}_{8}(\mathrm{Bi}-2212)$ for non-zero values of $T$ and $H$ is taken up in Section 5.1 (for $j=0$ ) and Section 5.2 (for $j \neq 0$ ). In Section 5.3 we present the solutions of these equations in the scenario of 1 PEM due to each of the ion species: $\mathrm{Ca}, \mathrm{Sr}$, and Bi. Sections 6 and 7 are devoted, respectively, to a Discussion of our findings and Conclusions. Finally, for the reader's convenience, we have given in an Appendix the conversion factors needed to go over from the natural system of units to the more familiar units employed in the BCS theory.

\section{Pairing Equation Incorporating Fermi Energy, Temperature and Applied Field When the Momentum of Cooper Pairs Is Zero}

Incorporating $T$ and $H$ into the pairing equation is a two-step process [17]: 1) temperature-generalization of the $T=0$ Bethe-Salpeter Equation (BSE) via the Matsubara recipe and 2) further generalization of the equation obtained in (1) to include $H$ via the Landau quantization scheme.

For elemental SCs for which pairing arises from the one-phonon exchange mechanism (1PEM) due to a single species of ions, Step (1) leads to

$$
1=\frac{V}{(2 \pi)^{3}} \frac{1}{2} \int_{E_{F}-k \theta}^{E_{F}+k \theta} \mathrm{d}^{3} p \frac{\tanh \left[(\beta / 2)\left(p^{2} / 2 m-E_{F}-W / 2\right)\right]}{p^{2} / 2 m-E_{F}-W / 2},
$$

where $(-V) \neq 0$ in a narrow region of $\pm k \theta$ around the Fermi surface is the model BCS interaction parameter, $\beta=1 / k T, k$ is the Boltzmann constant, $\theta$ the Debye temperature of the ions, and $W$ is one-half the binding energy of a pair which is to be identified with $\Delta$. The units employed are: $\mathrm{eV}, \hbar=c=1$, generally. However, for the convenience of the reader, in all the final equations that are actually employed in our calculations, the factors of $\hbar$ and $c$ have been made explicit.

If the field $H$ is applied in the $\mathrm{z}$-direction, then Step (2) consists of making the following substitutions in (1):

$$
\begin{aligned}
& \int \mathrm{d} p_{x} \mathrm{~d} p_{y}=2 \pi e H \sum_{n}, \frac{p_{x}^{2}}{2 m}+\frac{p_{y}^{2}}{2 m}=(n+1 / 2) \hbar \Omega_{1}(H) \\
& \Omega_{1}(H)=\frac{e H}{m^{*} c}=\Omega_{0} H / s, \\
& \Omega_{0}=e / m_{e} c=1.7588 \times 10^{7} \mathrm{rad} \cdot \mathrm{sec}^{-1} \cdot \mathrm{G}^{-1},\left(s=m^{*} / m_{e}\right)
\end{aligned}
$$

where $\Omega_{0}(H)$ is the cyclotron frequency corresponding to the free electron mass and we assume that $m^{*}=m_{e}$ when $j_{c}=0$. The transverse components of momentum are thus quantized into Landau levels and we have (1) as 


$$
1=\frac{e H V}{8 \pi^{2}} \int_{-L}^{L} \mathrm{~d} p_{z} \sum_{n=0}^{n_{m}} \frac{\tanh \left[(\beta / 2)\left(p_{z}^{2} / 2 m^{*}-E_{F}+(n+1 / 2) \hbar \Omega_{1}(H)-W / 2\right)\right]}{p_{z}^{2} / 2 m^{*}-E_{F}+(n+1 / 2) \hbar \Omega_{1}(H)-W / 2}
$$

where $L$ and $n_{m}$ are usually $\infty$. Since the energy of an electron in our problem is constrained to lie in a narrow shell around the Fermi surface, we fix $L$ and $n_{m}$ by appealing to the law of equipartition of energy and split the region in which $V \neq$ 0 as

$$
\begin{aligned}
-\frac{2 k \theta}{3} & \leq \frac{p_{x}^{2}+p_{y}^{2}}{2 m^{*}}=(n+1 / 2) \hbar \Omega_{1}(H) \leq \frac{2 k \theta}{3} \\
-\frac{k \theta}{3} & \leq \frac{p_{z}^{2}}{2 m^{*}}-E_{F} \leq \frac{k \theta}{3} .
\end{aligned}
$$

In terms of the variable $\xi=p_{z}^{2} / 2 m^{*}-E_{F}, \mathrm{~d} p_{z}=(1 / 2) \sqrt{2 m^{*} /\left(E_{F}+\xi\right)} \mathrm{d} \xi$ is given by

$$
\begin{aligned}
1= & \frac{e H V}{16 \pi^{2}} \sqrt{\frac{2 m^{*}}{E_{F}}} \int_{-L_{1}(\theta)}^{L_{1}(\theta)} \frac{\mathrm{d} \xi}{\sqrt{1+\xi / E_{F}}} \\
& \times \sum_{n=0}^{n_{m}(\theta, H)} \frac{\tanh \left[(\beta / 2)\left(\xi+(n+1 / 2) \hbar \Omega_{1}(H)-W / 2\right)\right]}{\xi+(n+1 / 2) \hbar \Omega_{1}(H)-W / 2}
\end{aligned}
$$

where

$$
L_{1}(\theta)=k \theta / 3
$$

and

$$
n_{m}(\theta, H)=\text { floor }\left\{\frac{2 k \theta}{3 \hbar \Omega_{1}(H)}-\frac{1}{2}\right\} \text {. }
$$

Putting $W=0$ and rewriting (5) in terms of $T$ and the dimensionless variable $x=\xi / \hbar \Omega_{1}\left(H_{c}\right)$, we obtain the equation for the critical field $H_{c}$ at temperature $T$ (equivalently, the equation for the critical temperature $T_{c}$ at field $H$ ) as

$$
\begin{aligned}
1= & \lambda_{m} \int_{-L_{2}\left(\theta, H_{c}\right)}^{L_{2}\left(\theta, H_{c}\right)} \frac{\mathrm{d} x}{\sqrt{1+\hbar \Omega_{1}\left(H_{c}\right) x / E_{F 1}}} \\
& \times \sum_{n=0}^{n_{m 2}\left(\theta, H_{c}\right)} \frac{\tanh \left[\left(\hbar \Omega_{1}\left(H_{c}\right) / 2 k T_{c}\right)(x+n+1 / 2)\right]}{x+n+1 / 2}
\end{aligned}
$$

where $E_{F}$ has been relabeled as $E_{F 1}$ in order to distinguish it from the $E_{F}$ that occurs in the equations when $\boldsymbol{P} \neq 0$,

$$
\begin{gathered}
L_{2}\left(\theta, H_{c}\right)=k \theta /\left[3 \hbar \Omega_{1}\left(H_{c}\right)\right], \\
n_{m 2}\left(\theta, H_{c}\right)=\text { floor }\left[2 L_{2}\left(\theta, H_{c}\right)-1 / 2\right]
\end{gathered}
$$

and

$$
\lambda_{m}\left(H_{c}, V\right)=\frac{e H_{c} V}{16 \pi^{2}} \sqrt{\frac{2 m^{*}}{E_{F 1}}} .
$$

Incorporating $T, H$ and $E_{F}$ (7) is the equation we had set out to obtain; it reduces to Equation (16) of [17] when $E_{F} \gg k \theta$. The integrand in (7) is mani- 
festly dimensionless. In Appendix A, we provide the necessary conversion factors which show that $\lambda_{m}$ too is dimensionless and enable one to employ in our framework the more familiar BCS units, i.e., $\mathrm{eV}-\mathrm{cm}^{3}$ for $V$ and Gauss for $H$.

\section{Pairing Equation Incorporating Fermi Energy, Temperature and Applied Field When the Momentum of Cooper Pairs Is Non-Zero}

It is a tenet of the BCS theory that the same interaction parameter $\lambda$ occurs in both the equation for $\left\{T=T_{c}, \Delta=0\right\}$ and the equation for $\left\{T=0, \Delta_{0} \neq 0\right\}$, where $\Delta_{0}$ is the gap at $T=0$. Similarly, we assume here that $\lambda_{m}$ remains unchanged when we go over from the $\boldsymbol{P}=0$ to $\boldsymbol{P} \neq 0$ equations. We now draw attention to the fact that in [15] where we dealt with the $\Delta_{0} s$ and $j_{c} s$ of various SCs, it was assumed that $E_{F}$ too has the same value in both the equation for $\Delta_{0}(H=0)$ and the equation for $P_{d} j_{c}$--a plausible justification for which being that we were dealing with the $j_{c} s$ too at $T=0$ and $H=0$. For the sake of generality, we now assume that the value of $E_{F}$ when $\boldsymbol{P} \neq 0$ is different from its value when $\boldsymbol{P}=0$. Hence, $E_{F}$ is labelled as $E_{F 2}$ in the present section.

The $T$ - and $\boldsymbol{P}$-dependent equation for pairing in the 1PEM scenario is [18]:

$$
1=\frac{V}{16 \pi^{2}} \int_{\ell}^{u} \mathrm{~d}^{3} p \frac{\tanh [\beta C(\boldsymbol{p}) / 2]+\tanh [\beta D(\boldsymbol{p}) / 2]}{C(\boldsymbol{p})+D(\boldsymbol{p})},
$$

where

$$
\begin{aligned}
& C(\boldsymbol{p})=\frac{(\boldsymbol{P} / 2+\boldsymbol{p})^{2}}{2 m^{*}}-E / 2=\frac{P^{2}}{8 m^{*}}+\frac{|\boldsymbol{P} \| \boldsymbol{p}| \cos (\boldsymbol{P}, \boldsymbol{p})}{2 m^{*}}+\frac{p^{2}}{2 m^{*}}-E_{F 2}-W / 2 \\
& D(\boldsymbol{p})=\frac{(\boldsymbol{P} / 2-\boldsymbol{p})^{2}}{2 m^{*}}-E / 2=\frac{P^{2}}{8 m^{*}}-\frac{|\boldsymbol{P} \| \boldsymbol{p}| \cos (\boldsymbol{P}, \boldsymbol{p})}{2 m^{*}}+\frac{p^{2}}{2 m^{*}}-E_{F 2}-W / 2
\end{aligned}
$$

$V \neq 0$ in the region

$$
E_{F 2}-k \theta \leq \frac{(\boldsymbol{P} / 2+\boldsymbol{p})^{2}}{2 m^{*}}, \frac{(\boldsymbol{P} / 2-\boldsymbol{p})^{2}}{2 m^{*}} \leq E_{F 2}+k \theta,
$$

and the total energy of a pair is $E=2 E_{F 2}+W$. When $\boldsymbol{P}=0$, (10) fixes the limits $\ell$ and $u$ in (9) as $\ell=E_{F 2}-k \theta$ and $u=E_{F 2}+k \theta$. When $\boldsymbol{P} \neq 0$, the lower limit in (9) follows from:

$$
E_{F 2}-k \theta \leq \frac{\left(\boldsymbol{P}_{c} / 2-\boldsymbol{p}\right)^{2}}{2 m^{*}}=p^{2} / 2 m^{*}-\left|P_{c}\right| p_{z} / 2 m^{*},
$$

where $\boldsymbol{P}_{c}$ is the critical momentum corresponding to $W=0, \quad p_{z}=|\boldsymbol{p}| \cos \left(\boldsymbol{P}_{c}, \boldsymbol{p}\right)$ and the $\boldsymbol{P}_{c}^{2}$-term in the expansion of $\left(\boldsymbol{P}_{c} / 2 \pm \boldsymbol{p}\right)^{2}$ has been neglected (as justified in [18]). Note that (11) automatically ensures that $E_{F 2}-k \theta<\left(\boldsymbol{P}_{c} / 2+\boldsymbol{p}\right)^{2} / 2 m^{*}$. Appealing to the law of equipartition of energy as earlier, we now assume that

$$
p_{z}^{2} / 2 m^{*} \cong E_{F 2} / 3, \text { i.e., } p_{z}=\sqrt{2 m^{*} E_{F 2} / 3},
$$

whence we have (11) as 


$$
\begin{aligned}
& -E_{1}(\theta)+E_{2}\left(E_{F 2}\right) \leq\left[\left(p_{z}^{2} / 2 m^{*}-E_{F 2}\right)+\left(p_{x}^{2}+p_{y}^{2}\right) / 2 m^{*}\right] \\
& \left(E_{1}(\theta)=k \theta, E_{2}\left(E_{F 2}\right)=P_{c} \sqrt{E_{F 2} / 6 m^{*}}\right) .
\end{aligned}
$$

which is now the lower limit in (9). Working out the upper limit similarly, we have the limits of (9) as:

$$
\begin{aligned}
-E_{1}(\theta)+E_{2}\left(E_{F 2}\right) & \leq\left[\left(p_{z}^{2} / 2 m^{*}-E_{F 2}\right)+\left(p_{x}^{2}+p_{y}^{2}\right) / 2 m^{*}\right] \\
& \leq E_{1}(\theta)-E_{2}\left(E_{F 2}\right) .
\end{aligned}
$$

Employing substitutions (2) in order to introduce $H$ into (9), we obtain

$$
\begin{aligned}
1= & \frac{e H_{c} V}{8 \pi^{2}} \int_{-\ell_{1}\left(\theta, E_{F 2}\right)}^{\ell_{1}\left(\theta, E_{F 2}\right)} \mathrm{d} p_{z} \\
& \times \sum_{n=0}^{n_{u 1}\left(\theta, E_{F 2}, H_{c}\right)} \frac{\tanh \left[A_{1}\left(T_{c}, H_{c}, E_{F 2}, p_{z}\right)\right]+\tanh \left[B_{1}\left(T_{c}, H_{c}, E_{F 2}, p_{z}\right)\right]}{2\left[\left(p_{z}^{2} / 2 m^{*}-E_{F 2}\right)+(n+1 / 2) \hbar \Omega_{2}\left(H_{c}\right)\right]}
\end{aligned}
$$

where

$$
\begin{gathered}
\Omega_{2}\left(H_{c}\right)=\Omega_{0} H_{c} / s \\
A_{1}\left(T_{c}, H_{c}, E_{F}, p_{z}\right)=\left(\frac{1}{2 k T_{c}}\right)\left[\left(\frac{p_{z}^{2}}{2 m^{*}}-E_{F 2}\right)+\left(n+\frac{1}{2}\right) \hbar \Omega_{2}\left(H_{c}\right)+E_{2}\left(E_{F 2}\right)\right] \\
B_{1}\left(T_{c}, H_{c}, E_{F}, p_{z}\right)=\left(\frac{1}{2 k T_{c}}\right)\left[\left(\frac{p_{z}^{2}}{2 m^{*}}-E_{F 2}\right)+\left(n+\frac{1}{2}\right) \hbar \Omega_{2}\left(H_{c}\right)-E_{2}\left(E_{F 2}\right)\right]
\end{gathered}
$$

and

$$
\begin{aligned}
& \ell_{1}\left(\theta, E_{F 2}\right)=(1 / 3)\left[E_{1}(\theta)-E_{2}\left(E_{F 2}\right)\right] \\
& n_{u 1}\left(\theta, E_{F 2}, H_{c}\right)=\text { floor }\left[2 \ell_{1}\left(\theta, E_{F 2}\right) / \hbar \Omega_{2}\left(H_{c}\right)-1 / 2\right] .
\end{aligned}
$$

We note that the factors of $(1 / 3)$ in the limits of the integral and $(2 / 3)$ in the upper limit of the sum occur because, appealing to the equipartition law, we have split the inequalities (13) as

$$
\begin{aligned}
(1 / 3)\left[-E_{1}(\theta)+E_{2}\left(E_{F 2}\right)\right] & \leq\left(p_{z}^{2} / 2 m^{*}-E_{F 2}\right) \leq(1 / 3)\left[E_{1}(\theta)-E_{2}\left(E_{F 2}\right)\right] \\
(2 / 3)\left[-E_{1}(\theta)+E_{2}\left(E_{F 2}\right)\right] & \leq\left[\left(p_{x}^{2}+p_{y}^{2}\right) / 2 m^{*}=(n+1 / 2) \hbar \Omega_{2}\left(H_{c}\right)\right] \\
& \leq(2 / 3)\left[E_{1}(\theta)-E_{2}\left(E_{F 2}\right)\right] .
\end{aligned}
$$

With

$$
\xi=p_{z}^{2} / 2 m^{*}-E_{F 2}, \mathrm{~d} p_{z}=\frac{1}{2} \sqrt{2 m^{*} /\left(E_{F 2}+\xi\right)} \mathrm{d} \xi
$$

(14) is transformed into

$$
\begin{aligned}
1= & \frac{\lambda_{m}\left(V, H_{c}\right)}{2} \int_{-\ell_{1}\left(\theta, E_{F 2}\right)}^{\ell_{1}\left(\theta, E_{F 2}\right)} \frac{\mathrm{d} \xi}{\sqrt{1+\xi / E_{F 2}}} \\
& \times \sum_{n=0}^{n_{u 1}\left(\theta, E_{F 2}, H_{c}\right)} \frac{\tanh \left[A_{2}\left(T, H_{c}, E_{F 2}, \xi\right)\right]+\tanh \left[B_{2}\left(T, H_{c}, E_{F 2}, \xi\right)\right]}{\xi+(n+1 / 2) \hbar \Omega_{2}\left(H_{c}\right)},
\end{aligned}
$$

where $\lambda_{m}\left(V, H_{c}\right)$ is given by $(8), A_{2}(.$.$) and B_{2}(.$.$) are given by A_{1}($.$) and B_{1}(.$.$) ,$ 
respectively, with $\left(p_{z}^{2} / 2 m^{*}-E_{F 2}\right)$ replaced by $\xi$. Employing the dimensionless variables $x=\xi / \hbar \Omega_{2}\left(H_{c}\right)$ and

$$
y=E_{1}(\theta) / E_{2}\left(E_{F 2}\right)=\frac{k \theta}{P_{c}} \sqrt{6 m^{*} / E_{F 2}},
$$

we finally obtain the desired equation incorporating $T, H$ and $E_{F 2}$ when $\boldsymbol{P} \neq 0$ in terms of $y$ as

$$
\begin{aligned}
1= & \frac{\lambda_{m}\left(V, H_{c}\right)}{2} \int_{-\ell_{2}\left(\theta, H_{c}, r, y\right)}^{\ell_{2}\left(\theta, H_{c}, r, y\right)} \frac{\mathrm{d} x}{\sqrt{1+\hbar \Omega_{2}\left(H_{c}\right) x / E_{F 2}}} \\
& \times \sum_{n=0}^{n_{u 2}\left(\theta, H_{c}, r, y\right)} \frac{\tanh \left[A_{3}(\ldots)\right]+\tanh \left[B_{3}(\ldots)\right]}{x+n+1 / 2},
\end{aligned}
$$

where

$$
\begin{gathered}
\ell_{2}\left(\theta, H_{c}, r, y\right)=\frac{E_{1}(\theta)(1-1 / r y)}{3 \hbar \Omega_{2}\left(H_{c}\right)} \\
n_{u 2}\left(\theta, H_{c}, r, y\right)=\text { floor }\left[2 \ell_{2}\left(\theta, H_{c}, r, y\right)-1 / 2\right] \\
A_{3}(\ldots)=A_{3}\left(\theta, T_{c}, H_{c}, r, y, x, n\right)=\left[\frac{\hbar \Omega_{2}\left(H_{c}\right)}{2 k T_{c}}\left\{x+n+1 / 2+\frac{E_{1}(\theta)}{r y \hbar \Omega_{2}\left(H_{c}\right)}\right\}\right] \\
B_{3}(\ldots)=B_{3}\left(\theta, T_{c}, H_{c}, r, y, x, n\right)=\left[\frac{\hbar \Omega_{2}\left(H_{c}\right)}{2 k T_{c}}\left\{x+n+1 / 2-\frac{E_{1}(\theta)}{r y \hbar \Omega_{2}\left(H_{c}\right)}\right\}\right] .
\end{gathered}
$$

Although the multiplier $r$ of $y$ in the above equations is unity for an elemental $\mathrm{SC}$, it has been introduced for later convenience when we deal with a composite SC characterized by multiple $\theta$ s. Taking the example of Bi-2212, we note that if the Debye temperature of the SC is $\theta_{0}$ and pairing takes place via the $\mathrm{Ca}$ or the $\mathrm{Sr}$ ions, then $r_{1}=\theta_{\mathrm{Ca}} / \theta_{0}=1$ (because $\theta_{\mathrm{Ca}}=\theta_{0}$ ) for the former case and $r_{2}=\theta_{\mathrm{Sr}} / \theta_{0} \neq 1$ (because $\theta_{\mathrm{Sr}}$ differs from $\theta_{0}$ ) for the latter.

\section{Equations for the Density of Superconducting Electrons, their Critical Velocity, Effective Mass, and Critical Current Density}

If we assume that (7) corresponding to a set of $\left\{E_{F 1}, T_{c 1}, H_{c 1}, j_{c 1}=0\right\}$-values and (19) corresponding to a set of $\left\{E_{F 2}, T_{C 2}, H_{c 2}, j_{c 2} \neq 0\right\}$-values have provided a value of $y$, then we are enabled to obtain the following equations for the density of superconducting electrons $n_{s}, P_{\mathcal{c}}$, the critical velocity $v_{c}$ and $j_{\mathcal{c}}$ where the requisite factors of $\hbar$ and $c$ have been inserted for the convenience of the reader.

$$
\begin{gathered}
n_{s}\left(s, E_{F 2}\right)=\frac{1}{3 \pi^{2}}\left(\frac{2 m^{*} E_{F 2}}{(\hbar c)^{2}}\right)^{3 / 2}=C_{1}\left(s E_{F 2}\right)^{3 / 2} \\
\left(C_{1}=\frac{\left(2 m_{e} c^{2}\right)^{3 / 2}}{3 \pi^{2}(\hbar c)^{3}} \cong 4.541 \times 10^{21}(\mathrm{eV})^{-3 / 2} \mathrm{~cm}^{-3}\right) \\
P_{c}\left(\theta, E_{F 2}, s, y\right)=k\left(6 s m_{e}\right)^{1 / 2} \frac{\theta}{y} E_{F 2}^{-1 / 2},
\end{gathered}
$$


whence

$$
\begin{gathered}
v_{c}\left(\theta_{0}, E_{F 2}, s, y\right)=\frac{c P_{c}}{2 s m_{e}}=C_{2} \frac{\theta_{0}}{y}\left(s E_{F 2}\right)^{-1 / 2} \\
\left(C_{2}=\frac{6^{1 / 2} c k m_{e}^{-1 / 2}}{2} \cong 4.426 \times 10^{3} \mathrm{eV}^{1 / 2} \mathrm{~K}^{-1} \mathrm{~cm} \mathrm{~s}^{-1}\right) \\
j_{c}\left(\theta_{0}, E_{F 2}, s, y\right)=e n_{s} v_{c}=C_{3} \frac{\theta_{0}}{y} s E_{F 2} \\
\left(C_{3}=\mathrm{eC}_{1} \mathrm{C}_{2}=\frac{2 \sqrt{3} e c k m_{e}}{3 \pi^{2}(\hbar c)^{3}} \cong 3.22 \times 10^{6} \mathrm{C} \mathrm{K}^{-1}(\mathrm{eV})^{-1} \mathrm{~cm}^{-2} \mathrm{~s}^{-1}\right)
\end{gathered}
$$

and

$$
s\left(\theta, E_{F 2}, j_{c}, r, y\right)=\frac{j_{c} r y}{C_{3} \theta E_{F 2}} .
$$

\section{5. $\mathrm{Bi}_{2} \mathrm{Sr}_{2} \mathrm{CaCu}_{2} \mathrm{O}_{8}(\mathrm{Bi}-2212)$}

\subsection{Pairing Equation in the 2PEM Scenario for Non-Zero Values of Temperature and Applied Field when the Critical Current Density Is Zero}

We now undertake a study of Bi-2212 for which $T_{c}=86 \mathrm{~K}$ when $H=0$. When $H \neq 0$, it is characterized by [19]

$$
T_{c 1}=65 \mathrm{~K}, H_{c 1}=36 \text { Tesla, } j_{c 1}=0 .
$$

Among the 14 different values of $j_{c}$ that have been reported for the same SC at different values of $T$ and $H$, we focus here on the following two which are particularly interesting because they correspond to the same values of $T$ and $H$ :

$$
\begin{gathered}
T_{c 2}=4.2 \mathrm{~K}, H_{c 2}=12 \text { Tesla, } j_{c 2}=2.4 \times 10^{5} \mathrm{~A} / \mathrm{cm}^{2} \\
T_{c 3}=T_{c 2}, H_{c 3}=H_{c 2}, j_{c 3}=1.0 \times 10^{6} \mathrm{~A} / \mathrm{cm}^{2} .
\end{gathered}
$$

For the data in (28), we need to employ (7) which corresponds to $j_{c}=0$, whereas for the data in (29) and (30) we need (19). In either case, first of all, we require the value of the Debye temperature $\theta_{0}$ of the SC, which is $237 \mathrm{~K}$. Since we are now dealing with a composite SC [(7) was derived for an elemental SC], we have the following multiple Debye temperatures at play because of the anisotropy of the SC [20]:

$$
\begin{aligned}
& \theta(\mathrm{Bi}-2212)=\theta_{0}=237 \mathrm{~K} \\
& \theta_{\mathrm{Ca}}=237, \theta_{\mathrm{Sr}}=286 \mathrm{~K}, \theta_{\mathrm{Bi}}=269 \mathrm{~K} .
\end{aligned}
$$

Note that $y$ in (18) is defined in terms of the Debye temperature of the SC (not in terms of the Debye temperature of any of the constituents of the SC if it consists of several elements). Sticking to the same definition, we have

$$
y(\operatorname{Bi}-2212)=E_{1}\left(\theta_{0}\right) / E_{2}\left(E_{F 2}\right)=\frac{k \theta_{0}}{P_{c}} \sqrt{\frac{6 s m_{e}}{E_{F 2}}},
$$

and, in all the four equations following (19), we need to have [15] $r=\theta_{\mathrm{Ca}} / \theta_{0}$, 
$\theta_{\mathrm{Sr}} / \theta_{0}$ and $\theta_{\mathrm{Bi}} / \theta_{0}$ for pairing via the $\mathrm{Ca}, \mathrm{Sr}$ and $\mathrm{Bi}$ ions, respectively.

Following our study dealing with the $T_{\mathcal{c}} s, \Delta_{0} s$ and $j_{0} s$ of Bi-2212 reported in [20], it seems imperative that we generalize (7) and (19) to the case of pairing via the 2PEM scenario. To this end, it is convenient to define

$$
\begin{gathered}
L_{3}\left(\theta, H_{c}\right)=\frac{k \theta}{3 \hbar \Omega_{1}\left(H_{c}\right)}, n_{u 3}\left(\theta, H_{c}\right)=\text { floor }\left[2 L_{3}\left(\theta, H_{c}\right)-1 / 2\right] \\
\phi_{1}\left(H_{c}, E_{F}, x\right)=1 / \sqrt{1+\hbar \Omega_{1}\left(H_{c}\right) x / E_{F}} \\
F_{1}\left(\theta, T_{c}, H_{c}, x\right)=\sum_{n=0}^{n_{u 3}\left(\theta, H_{c}\right)} \frac{\tanh \left[\frac{\hbar \Omega_{1}\left(H_{c}\right)}{2 k T_{c}}(x+n+1 / 2)\right]}{x+n+1 / 2} \\
G_{1}\left(\theta, T_{c}, H_{c}, E_{F 1}\right)=\int_{-L_{3}\left(\theta, H_{c}\right)}^{L_{3}\left(\theta, H_{c}\right)} d x \phi_{1}\left(H_{c}, E_{F}, x\right) F_{1}\left(\theta, T_{c}, H_{c}, x\right),
\end{gathered}
$$

which lead to the following equation in the $2 \mathrm{PEM}$ scenario as a generalization of (7) for pairing via the $\mathrm{Ca}$ and $\mathrm{Sr}$ ions when $j_{c}=0$ :

$$
\begin{aligned}
& E q 1\left(\theta_{1}, \theta_{2}, T_{c 1}, H_{c 1}, E_{F 1}, \lambda_{m 1}, \lambda_{m 2}\right) \\
& \equiv \operatorname{Re}\left[1-\lambda_{m 1} G_{1}\left(\theta_{1}, T_{c 1}, H_{c 1}, E_{F 1}\right)-\lambda_{m 2} G_{1}\left(\theta_{2}, T_{c 1}, H_{c 1}, E_{F 1}\right)\right]=0 .
\end{aligned}
$$

For the $\theta$-values in (31), we note that (i) solution of (33) with inputs from (28) leads to (a) the value of $\lambda_{m 1}$ for pairing in the 1PEM scenario via the Ca ions by putting $\theta_{1}=\theta_{\mathrm{Ca}}, \lambda_{m 2}=0$ and (b) the value of $\lambda_{m 2}$ for pairing in the 1PEM scenario via the Sr ions by putting $\lambda_{m 1}=0, \theta_{2}=\theta_{\mathrm{Sr}}$ and (ii) the need to invoke $2 \mathrm{PEM}$ will arise only if either of the so-obtained $\lambda_{m} s$ exceeds the Bogoliubov limit of 0.5 , because beyond this value the system becomes unstable.

\subsection{Pairing Equation in the 2PEM Scenario for Non-Zero Values of Temperature, Applied Field and Critical Current Density}

If we obtain the generalized version of (19) to deal with pairing in the 2PEM scenario following the same procedure as above, then with the inputs from (29) and (30) we have two equations besides (33). These three equations are not sufficient to deal with at least the six unknowns in our problem: $\lambda_{m 1}, \lambda_{m 2}, E_{F 1}, E_{F 2}$, $E_{F 3}$ and $y$. Therefore, ab-initio calculation of the $j_{\mathcal{C}} s$ via this approach will involve making ad hoc assumptions about some of the unknowns. To avoid such a situation, we adopt the strategy of incorporating in the generalized version of (19) the empirical $j_{c}$-values noted in (29) or (30). Even though the number of equations in this approach continues to be three, we show below how it sheds light on several important features of the data in (29) and (30).

In order to incorporate $j_{c}$ into (19), we employ (27) for $s\left(\theta, E_{F}, j_{\mathcal{O}} r, y\right)$, whence we have (15) as

$$
\Omega_{2}\left(H_{c}\right) \rightarrow \Omega_{2}\left(\theta, H_{c}, E_{F}, j_{c}, r, y\right)=\frac{C_{3} \Omega_{0} H_{c} \theta E_{F}}{j_{c} r y}
$$

and (20)-(23) as 


$$
\begin{gathered}
\ell_{4}\left(H_{c}, E_{F}, j_{c}, r, y\right)=\frac{k j_{c}(r y-1)}{3 C_{3} \hbar \Omega_{0} H_{c} E_{F}} \\
n_{u 4}\left(H_{c}, E_{F}, j_{c}, r, y\right)=\text { floor }\left[2 \ell_{4}\left(H_{c}, E_{F}, j_{c}, r, y\right)-1 / 2\right] \\
A_{3}(\ldots)=A_{3}\left(\theta, T_{c}, H_{c}, E_{F}, j_{c}, r, y, x, n\right) \\
=\left[\frac{\hbar \Omega_{2}\left(\theta, H_{c}, E_{F}, j_{c}, r, y\right)}{2 k T_{c}}\left\{x+n+\frac{1}{2}+\frac{k \theta}{\hbar \Omega_{2}\left(\theta, H_{c}, E_{F}, j_{c}, r, y\right)}\right\}\right] \\
B_{3}(\ldots)=B_{3}\left(\theta, T_{c}, H_{c}, E_{F}, j_{c}, r, y, x, n\right) \\
=\left[\frac{\hbar \Omega_{2}\left(\theta, H_{c}, E_{F}, j_{c}, r, y\right)}{2 k T_{c}}\left\{x+n+1 / 2-\frac{k \theta}{\hbar \Omega_{2}\left(\theta, H_{c}, E_{F}, j_{c}, r, y\right)}\right\}\right] .
\end{gathered}
$$

With the definitions

$$
\begin{gathered}
\phi_{2}\left(\theta, H_{c}, E_{F}, j_{c}, x, r, y\right)=\frac{1}{\sqrt{1+\hbar \Omega_{2}\left(\theta, H_{c}, E_{F}, j_{c}, r, y\right) x / E_{F}}} \\
F_{2}\left(\theta, T_{c}, H_{c}, E_{F}, j_{c}, x, r, y\right)=\sum_{n=0}^{n_{u 4}\left(H_{c}, E_{F}, j_{c}, r, y\right)} \frac{A_{3}(\ldots)+B_{3}(\ldots)}{x+n+1 / 2},
\end{gathered}
$$

where $\ell_{4}(\ldots), n_{u 4}(\ldots), A_{3}(\ldots)$ and $B_{3}(\ldots)$ are given by (34), (35), (36) and (37), respectively, and

$$
G_{2}\left(\theta, T_{c}, H_{c}, E_{F}, j_{c}, r, y\right)=\int_{-\ell_{4}(\ldots)}^{\ell_{4}(\ldots)} \mathrm{d} x \phi_{2}(\ldots) F_{2}(\ldots),
$$

we obtain, in the 2PEM scenario as a generalization of (19) for pairing via two species of ions and a given value of $j_{\mathcal{c}}$ the following equation:

$$
\begin{aligned}
& \operatorname{Eq} 2\left(\theta_{1}, \theta_{2}, T_{c}, H_{c}, q, E_{F}, j_{c}, r_{1}, r_{2}, y, \lambda_{m 1}, \lambda_{m 2}\right) \\
& \equiv \operatorname{Re}\left[1-\lambda_{m 1} G_{2}\left(\theta_{1}, T_{c}, H_{c}, E_{F}, j_{c}, r_{1}, y\right)-\lambda_{m 2} G_{2}\left(\theta_{2}, T_{c}, H_{c}, E_{F}, j_{c}, r_{2}, y\right)\right]=0 .
\end{aligned}
$$

\subsection{Solutions in the Scenario of 1PEM Due to Either of the Ca, Bi and the Sr Ions}

In order to unravel the empirical features of Bi-2212 noted in (28), (29) and (30) in the above framework, we proceed as follows:

1) We first deal with the data in (28) via (33) where, in the latter equation, $E_{F I}$ is an independent variable. If we solve this equation for different assumed values of $E_{F 1}=\rho k \theta_{0}$ and $\lambda_{m 2}=0$, we obtain the corresponding values of $\lambda_{m 1}$ for pairing via the Ca ions. We thus find that for $\rho=10, \lambda_{m 1}=0.31283$ and for all values of $\rho>10, \lambda_{m 1}$ has the same value up to four significant digits. For the same value of $\rho$ and $\lambda_{m 1}=0$, the corresponding value of $\lambda_{m 2}(\mathrm{Bi})$ and $\lambda_{m 2}(\mathrm{Sr})$ via (33) are 0.22043 and 0.20778 , respectively Similarly obtained values of $\lambda_{m 1}(\mathrm{Ca}), \lambda_{m 2}(\mathrm{Bi})$ and $\lambda_{m 2}(\mathrm{Sr})$ for some select values of $\rho \leq 10$ are given in Table 1 .

2) Since none of the values of $\lambda_{m}$ in Table 1 exceeds the Bogoliubov limit of 0.5 , we now employ (39) also in the $1 \mathrm{PEM}$ scenario in order to determine $E_{F 2}$ corresponding to the data in (29). To this end for $\rho=10$, for pairing via the $\mathrm{Ca}$ ions, we use the following values in (39): 
Table 1. Obtained via (33), the interaction parameters $\lambda_{m 1}(\mathrm{Ca}), \lambda_{m 2}(\mathrm{Bi})$ and $\lambda_{m 2}(\mathrm{Sr})$ for pairing via the $\mathrm{Ca}, \mathrm{Bi}$, and $\mathrm{Sr}$ ions corresponding to $T_{c 1}=65 \mathrm{~K}, H_{c 1}=36 \times 10^{4} \mathrm{G}$ with the additional inputs $\left\{\lambda_{m 2}=0, \theta_{1}=237\right\},\left\{\lambda_{m 1}=0, \theta_{2}=269 \mathrm{~K}\right\}$ and $\left\{\lambda_{m 1}=0, \theta_{2}=286 \mathrm{~K}\right\}$, respectively.

\begin{tabular}{clll}
\hline$\rho=\frac{E_{F 1}}{k \theta_{0}}$ & $\lambda_{m 1}(\mathrm{Ca})$ & $\lambda_{m 2}(\mathrm{Bi})$ & $\lambda_{m 2}(\mathrm{Sr})$ \\
\hline 10 & 0.31283 & 0.22043 & 0.20778 \\
5 & 0.31243 & 0.22003 & 0.20736 \\
4 & 0.31219 & 0.21980 & 0.20712 \\
3 & 0.31176 & 0.21937 & 0.20667 \\
2 & 0.31069 & 0.21835 & 0.20558 \\
1 & 0.30594 & 0.21382 & 0.20075 \\
\hline
\end{tabular}

$$
\begin{aligned}
& \lambda_{m 2}=0, \lambda_{m 1}=0.31283 ; T_{c}=T_{c 2}, H_{c}=H_{c 2}, j_{c}=j_{c 2} ; \\
& r=r_{1}=1, E_{F}=q E_{F 1}\left(E_{F 1}=\rho k \theta_{0}\right),
\end{aligned}
$$

which leaves out $y$ which is yet to be specified. Since $y$ too is an independent variable, we need to solve (39) for a range of values of $r y>1$; that $r y$ must be greater than unity follows from (34). We find that for each such value of $y$, the solution of the transcendental Equation (39) yields, in general, multiple roots each of which corresponds not only to $j_{c 2}$ (which is an in-built feature of our formalism), but also, to the accuracy with which they are quoted in Table 2 and Table 3, the same values for $n_{s}$ and $v_{c}$ via (24) and (25), respectively. For this reason, corresponding to each $y$, given in Table 2 are only the greatest roots of (39), which are found by first plotting (39) against $q$ to determine the range outside which the equation does not have any roots.

In the context of the critical current densities, such a plot given in Figure 1 seems to be unusual and will be discussed below. The largest value of $q$ corresponding to any value of $y$ at which the function being plotted crosses zero is then found more accurately using numerical root-finding methods and is given in Table 2 where similar results for $\rho=5$ and 1 for both pairings via the $\mathrm{Ca}$ and the $\mathrm{Sr}$ ions are also given.

The parameters, the values of which are different for different roots, corresponding to the same value of y are $n_{u 4}, s$ and $E_{F 2}$ where $n_{u 4}$ and $s$ are determined via (35) and (27), respectively, and $E_{F 2}=q E_{F 1}$. Of these, the values of $s$ dictate the range of $y$ relevant for the data under consideration. To elaborate, for $y \leq 2.40$, it is found that $s \geq 37$. Since, contrary to the known features of the SC under consideration, such values of $s$ would put it in the category of heavy-fermion SCs [13], they must be excluded and so we set the lower limit of $y$ at a value which leads to $s \approx 10$. The value of $s$ decreases as $y$ is progressively increased. This feature enables us to set the upper limit on the value of $y$ as one for which $s \approx 1$.

3) For the data in (30), we also give in Table 3 the results that follow when the operative 1PEM is due to the Sr ions. 
Table 2. The largest values of $q \equiv E_{F 2} / E_{F 1}$ corresponding to $y_{1}$ and $y_{2}$ obtained by solving (39) for the set $S_{1}=\left\{T_{\mathcal{c}}, H_{\mathcal{c}}, j_{\mathcal{c}}\right.$ ion species $\}$ as noted and different pairs of $S_{2}=\left\{\rho, \lambda_{m}\right\}$ values taken from Table 1. $n_{u 4}, s, n_{s}$ and $v_{c}$ corresponding to $q_{1}$ and $q_{2}$ are calculated via (35), (27), (24) and (25), respectively.

\begin{tabular}{|c|c|c|c|c|c|c|c|}
\hline \multicolumn{8}{|c|}{$T_{c}=T_{c 2}=4.2 \mathrm{~K}, H_{c}=H_{c 2}=12 \times 10^{4} \mathrm{G}, j_{c}=j_{c 2}=2.4 \times 10^{5} \mathrm{~A} \cdot \mathrm{cm}^{-2}$, Pairing via Ca ions } \\
\hline$\rho, \lambda$ & $\begin{array}{l}y_{1}, y_{2} \text { as in } \\
y_{1} \leq y \leq y_{2}\end{array}$ & $\begin{array}{l}q_{1}, q_{2} \text { as in } \\
q_{1} \leq q \leq q_{2} \\
\left(\times 10^{-4}\right)\end{array}$ & $\begin{array}{l}n_{u 1}, n_{u 2} \text { as in } \\
n_{1} \geq n_{u 4} \geq n_{2}\end{array}$ & $\begin{array}{l}s_{1}, s_{2} \text { as in } \\
s_{1} \geq s \geq s_{2}\end{array}$ & $\begin{array}{l}n_{s 1}, n_{s 2} \text { as in } \\
n_{s 1} \leq n_{s} \leq n_{s 2} \\
\left(\times 10^{17} \mathrm{~cm}^{-3}\right)\end{array}$ & $\begin{array}{l}v_{c 1}, v_{c 2} \text { as in } \\
v_{c 1} \geq v_{c} \geq v_{c 2} \\
\left(\times 10^{7} \mathrm{~cm} / \mathrm{s}\right)\end{array}$ & $\begin{array}{l}E_{F 2}^{1}=q_{1} E_{F 1} \\
E_{F 2}^{2}=q_{2} E_{F 1} \\
E_{F 2}^{1} \leq E_{F 2} \leq E_{F 2}^{2} \\
\left(\times 10^{-4} \mathrm{eV}\right)\end{array}$ \\
\hline $10,0.31283$ & $2.575,3.10$ & $4.5194,48.041$ & 52,6 & $8.77,0.99$ & $1.05,1.38$ & $1.43,1.08$ & $0.92,9.81$ \\
\hline $5,0.31243$ & $2.575,3.10$ & $9.0422,96.168$ & 52,6 & $8.77,0.99$ & $1.05,1.38$ & $1.43,1.08$ & $0.92,9.82$ \\
\hline $1,0.30594$ & $2.575,3.20$ & $43.589,472.32$ & 54,6 & $9.10,1.04$ & $1.05,1.45$ & $1.43,1.03$ & $0.89,9.65$ \\
\hline \multicolumn{8}{|c|}{$T_{c}=T_{c 2}=4.2 \mathrm{~K}, H_{c}=H_{c 2}=12 \times 10^{4} \mathrm{G}, j_{c}=j_{c 2}=2.4 \times 10^{5} \mathrm{~A} \cdot \mathrm{cm}^{-2}$, Pairing via Sr ions } \\
\hline $10,0.20778$ & $2.50,3.35$ & $3.9111,49.813$ & 77,8 & 9.841 .04 & $1.00,1.55$ & $1.50,0.96$ & $0.80,10.2$ \\
\hline $5,0.2076$ & $2.50,3.35$ & $7.9476,99.83$ & 76,8 & $9.69,1.03$ & $1.00,1.55$ & $1.50,0.96$ & $0.81,10.2$ \\
\hline $1,0.20075$ & $2.51,3.35$ & $39.478,516.30$ & 77,8 & $9.79,1.00$ & $1.01,1.55$ & $1.49,0.96$ & $0.81,10.5$ \\
\hline
\end{tabular}

Table 3. The largest values of $q \equiv E_{F 2} / E_{F 1}$ corresponding to $y_{1}$ and $y_{2}$ obtained by solving (39) for the set $S_{1}=\left\{T_{\mathcal{c}}, H_{\mathcal{c}} j_{\mathcal{c}}\right.$ ion species $\}$ as noted and different pairs of $S_{2}=\left\{\rho, \lambda_{m}\right\}$ values taken from Table 1. $n_{u 4}, s, n_{s}$ and $v_{c}$ corresponding to $q_{1}$ and $q_{2}$ are calculated via (35), (27), (24) and (25), respectively.

$$
T_{c}=T_{c 3}=4.2 \mathrm{~K}, H_{c}=H_{c 3}=12 \times 10^{4} \mathrm{G}, j_{c}=j_{c 3}=1.0 \times 10^{6} \mathrm{~A} \cdot \mathrm{cm}^{-2} \text {, Pairing via Ca ions }
$$

\begin{tabular}{|c|c|c|c|c|c|c|c|}
\hline$\rho, \lambda$ & $\begin{array}{l}y_{1}, y_{2} \text { as in } \\
y_{1} \leq y \leq y_{2}\end{array}$ & $\begin{array}{l}q_{1}, q_{2} \text { as in } \\
q_{1} \leq q \leq q_{2} \\
\left(\times 10^{-3}\right)\end{array}$ & $\begin{array}{l}n_{u 1}, n_{u 2} \text { as in } \\
n_{1} \geq n_{u 4} \geq n_{2}\end{array}$ & $\begin{array}{l}s_{1}, s_{2} \text { as in } \\
s_{1} \geq s \geq s_{2}\end{array}$ & $\begin{array}{l}n_{s 1}, n_{s 2} \text { as in } \\
n_{s 1} \leq n_{s} \leq n_{s 2} \\
\left(\times 10^{17} \mathrm{~cm}^{-3}\right)\end{array}$ & $\begin{array}{l}v_{c 1}, v_{c 2} \text { as in } \\
v_{c 1} \geq v_{c} \geq v_{c 2} \\
\left(\times 10^{6} \mathrm{~cm} / \mathrm{s}\right)\end{array}$ & $\begin{array}{l}E_{F 2}^{1}=q_{1} E_{F 1} \\
E_{F 2}^{2}=q_{2} E_{F 1} \\
E_{F 2}^{1} \leq E_{F 2} \leq E_{F 2}^{2} \\
\left(\times 10^{-4} \mathrm{eV}\right)\end{array}$ \\
\hline $10,0.31283$ & $2.40,2.90$ & $1.5499,17.720$ & 56,6 & $9.94,1.05$ & $8.01,10.6$ & $7.79,5.87$ & $3.17,36.2$ \\
\hline $5,0.31243$ & $2.40,2.90$ & $3.2281,35.461$ & 54,6 & $9.54,1.05$ & $8.01,10.6$ & $7.79,5.87$ & $3.30,36.2$ \\
\hline $1,0.30594$ & $2.40,2.90$ & $15.274,179.10$ & 57,6 & $10.08,1.04$ & $8.01,10.6$ & $7.79,5.87$ & $3.12,36.6$ \\
\hline \multicolumn{8}{|c|}{$T_{c}=T_{c 3}=4.2 \mathrm{~K}, H_{c}=H_{c 3}=12 \times 10^{4} \mathrm{G}, j_{c}=j_{c 3}=1.0 \times 10^{6} \mathrm{~A} \cdot \mathrm{cm}^{-2}$, Pairing via Sr ions } \\
\hline $10,0.20778$ & $2.30,2.90$ & $1.4543,20.791$ & 76,7 & $10.15,0.89$ & $7.51,10.6$ & $8.31,5.87$ & $2.97,42.5$ \\
\hline $5,0.20736$ & $2.30,2.90$ & $2.9099,35.048$ & 76,8 & $10.14,1.06$ & $7.51,10.6$ & $8.31,5.87$ & $2.97,35.8$ \\
\hline $1,0.20075$ & $2.31,2.90$ & $14.619,177.80$ & 76,8 & $10.14,1.05$ & $7.56,10.6$ & $8.25,5.87$ & $2.99,36.3$ \\
\hline
\end{tabular}

4) Pairing in Bi-2212 can of course also take place via the Bi ions in the 1PEM scenario. From the results obtained via the $\mathrm{Ca}$ and the $\mathrm{Sr}$ ions and the value of $\theta_{\mathrm{Bi}}$, which lies between $\theta_{\mathrm{Ca}}$ and $\theta_{\mathrm{Sr}}$ [vide (31)], one would expect that $\lambda_{m}(\mathrm{Bi})$ for any of the value of $\rho$ in either of the two Tables should lie between the corresponding values of $\lambda_{m}(\mathrm{Ca})$ and $\lambda_{m}(\mathrm{Sr})$ and that, but for this change, the overall results should be substantially similar to those already obtained. This is indeed found to be so, as can be seen from the following results corresponding to $T=$ $4.2 \mathrm{~K}, H_{c}=12 \times 10^{4} \mathrm{G}$ and $j_{c}=2.4 \times 10^{5} \mathrm{~A} / \mathrm{cm}^{2}$ as an example: 


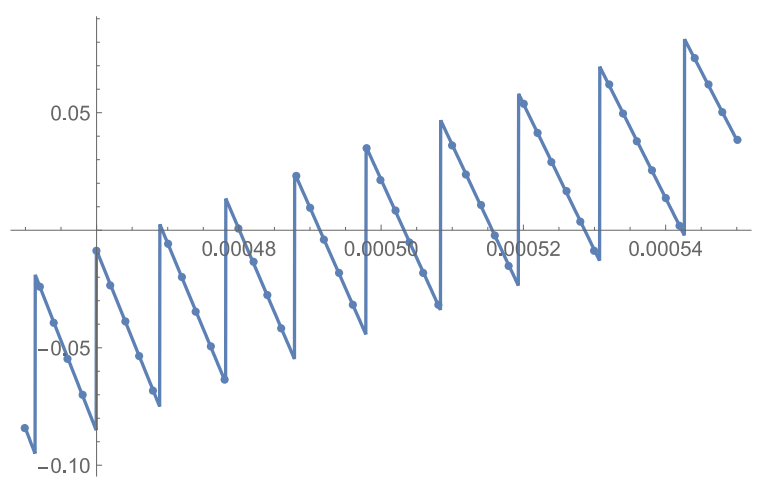

Figure 1. Plot of Eq $2\left(\theta_{1}, \theta_{2}, T_{c 2}, H_{c 2}, q, E_{F 1}, j_{c 2}, r_{1}, r_{2}, y, \lambda_{m 1}, 0\right)$ vide (39) as a function of $q$ showing multiple roots of (39) in the indicated range corresponding to $\theta_{1}=237, r_{1}=1, \rho$ $=10, \lambda_{m 1}=0.31283, y=2.60$ and $\left\{T_{c 2}, H_{c 2}, j_{c 2}\right\}$ as in (29).

$$
\begin{aligned}
& \rho=10, \lambda=0.22043 ; 2.54 \leq y \leq 3.30 \\
& 4.0226 \times 10^{-4} \leq q \leq 5.4521 \times 10^{-3}, 70 \geq n_{u} \geq 7,9.72 \geq s \geq 0.93 \\
& 1.03 \times 10^{17} \leq n_{s} \leq 1.52 \times 10^{17}, 1.46 \times 10^{7} \geq v_{c} \geq 9.87 \times 10^{6} \\
& 0.82 \times 10^{-4} \leq E_{F 2} \leq 11.1 \times 10^{-4},
\end{aligned}
$$

where the units for $n_{s}, V_{c}$ and $E_{F}$ are $\mathrm{cm}^{-3}, \mathrm{~cm} / \mathrm{s}$ and $\mathrm{eV}$, respectively.

\section{Discussion}

To give a perspective of the approach followed in this paper, it is pertinent to point out that, conventionally, the $j_{c}$ of an SC is determined via one or the other critical state models; for characteristic equations of nine critical state models, see ([19], p61). It is postulated in such models that for low applied fields or currents the outer part of the sample is in the so-called critical state which is characterized by particular values of $j_{c}$ and $H$ and that the interior of the SC is shielded from these fields and currents. For Bi-2212, the most commonly employed model is Bean's model where its $j_{c}$ is determined via the geometry of the sample and the magnetization width $\Delta M$ of the $M(H)$ hysteresis loop; see, e.g. [21], which gives values of $j_{c}(H)$ of the melt quenched and the non-melted samples of the SC at $5 \mathrm{~K}$. Following the conventional approach, the significantly different $j_{c}$ $(H)$-values of the samples in [21] are attributed to material properties of the samples such as their cell parameters, alignment and inter-connectivity of the grains and the grain boundaries. It is hence seen that the approach followed in our paper differs radically from the conventional approach.

A remark about the operator $R e$ in (33): The function $G_{1}\left(\theta_{1}, T_{c 1}, H_{c 1}, E_{F 1}\right)$ in this equation becomes pure imaginary for all values of $x<-E_{F} / \hbar \Omega_{1}\left(H_{c}\right)$ because of $\phi_{1}\left(H_{c 1}, E_{F 1}, x\right)$. This is a situation which also occurs in several other problems, e.g., while dealing with heavy-fermion SCs [13] and BCS-BEC crossover [22]. In order then to obtain real solutions, one alternative is to manually shift the lower limit of integration. This becomes cumbersome if one is simultaneously dealing with more than one such equation. The operator Re provides a much simpler, one-step, alternative, as was also noted in the context of Fe-based 
SCs [23]. This remark also applies to (39).

As was noted above, the solution of (39) leads to multiple roots for any value of $y$ for which $s$ falls in the range of our interest. These are shown in Figure 1 for a particular value of $y$. A notable feature of this figure is its saw-tooth appearance (a series of "Vs"), which is attributable to a combination of the fluctuations in Fermi energy and the floor function employed in our formalism. The unmarked vertical limbs of the Vs are discontinuities which occur when the summation index $n$ changes discretely from one integral value to another due to the floor function. We note that no root is found even when such limbs cross zero, as also that the saw-tooth behavior is not seen when (33) is solved despite the fact that it too employs the floor function, which is so because it is solved for a fixed value of $E_{F}$

Another feature of (39) is that as $y$ is progressively increased, the number of its roots keeps decreasing, as an example of which we note that as one goes from $y=2.60$ to 3.10 in the upper-half of Table 2, the number of roots changes from 7 to 2. This is reminiscent of Melde's experiment with stationary waves on a taut string clamped at both ends which are therefore nodal points. In-between these, the string displays a varying number of nodes depending on the frequency of the tuning fork with which it is induced to vibrate. One is thus led to surmise that: 1) (39) embodies content that leads to behavior akin to a Melde's string, 2) the variation of $y$ in the present case is equivalent to changing the frequency of the tuning fork and 3) the values of $q$ at which (39) vanishes (i.e., the roots of the equation) are equivalent to the nodes of the Melde's string.

Regarding the employment of $\Omega_{0}$ (which corresponds to free electron mass) in obtaining solutions of (33), we note that there is no loss of generality in making this assumption in so far as the solutions of this equation are employed to shed light on why the same SC at the same values of $T$ and $H$ sustains different values of $j_{c}$-which is the chief objective of this study. This assertion follows from the fact that different pairs of $\left\{\rho, \lambda_{m}\right\}$ in both Table 2 and Table 3 lead to essentially similar results for each of the following parameters: $n_{u 4}, s, n_{s}, v_{c}$ and $E_{F}$.

In so far as the $n_{u}$ values given in Table 2 and Table 3 are concerned, we recall from [17] that the radius of the largest Landau orbit is given by

$$
r_{n}(H)=r_{0} \sqrt{2 n_{u}(H)+1}
$$

where $r_{0}=\sqrt{\hbar c / e H_{0}}$ and $H_{0}$ is the critical applied field at $T=0$ and that $n_{u}\left(H_{0}\right)$ for the elemental SCs $\mathrm{Hg}$, In, $\mathrm{Tl}$ and Sn, for example, has the values 1062, 1900, 2201 and 3172, respectively. Since $r_{n}(H)$ and therefore $n_{u}$ may also be regarded as a measure of the coherence length of the SC, the low values of the latter in the two Tables signify that Bi-2212 has a much smaller coherence length than the elemental SCs, which is in accord with the known empirical facts.

If, for both the values of $j_{\mathcal{c}}$ i.e., $j_{c_{2}}=2.4 \times 10^{5}$ (for an Ag-sheathed tape) and $j_{c 3}=1.0 \times 10^{6} \mathrm{~A} / \mathrm{cm}^{2}$ (for a multilayer tape), the SC is assumed to have nearly the same value of $s$, which is unlikely, then the results in Table 2 and Table 3 show that 1) $\left.n_{s}\left(j_{c 2}\right)<n_{s}\left(j_{c 3}\right), 2\right) \quad v_{c}\left(j_{c 2}\right)>v_{c}\left(j_{c 3}\right)$ and 3) $E_{F}\left(j_{c 2}\right)<E_{F}\left(j_{c 3}\right)$. All 
of these results remain valid even if $s\left(j_{\mathrm{c} 2}\right) \approx 10$ and $s\left(j_{\mathrm{c} 3}\right) \approx 1$. However, if $s\left(j_{\mathrm{c} 2}\right) \approx 1$ and $s\left(j_{c 3}\right) \approx 10$ then, while the first two results still hold, we have $E_{F}\left(j_{c 2}\right)>E_{F}\left(j_{c 3}\right)$. In so far as the absolute values of $E_{F}$ in Table 2 and Table 3 are concerned, the lowest among them are of the order of $0.3 \mathrm{meV}$ or less; these are interpretable as being near the nodes or the node lines on the Fermi surface.

\section{Conclusions}

1) Based on the microscopic BSE customized to deal with superconductivity, we have given here derivation of equations that incorporate $E_{F}$ (equivalently, the chemical potential $\mu$ ), $T, H$, and $P$.

2) Among the main results of this paper are (33) and (39). The former generalizes the equation given in [17] that incorporated $T$ and $H$, but not $E_{\vec{F}}$ the latter generalizes the equation given in [18] that incorporated $T, E_{F}$ and $P$, but not $H$.

3) Another notable result of the approach followed here is that it sheds light on why the cuprate that we have dealt with has much smaller coherence length than elemental SCs.

4) A novel aspect of our work is that it incorporates $j_{\mathcal{o}}$, which is a sample-specific property, into the dynamical equations that govern pairing in the SC.

5) As is well-known, the $j_{c}$ of an SC depends on several factors such as its size, shape, and how it is doped and prepared. Based on the premise that the $E_{F}$ of an SC subsumes all of these features, we have given here a framework for testing it, and applied it for a detailed study of $\mathrm{Bi}-2212$.

6) The upshot of the present work is that for greater substantiation of the above premise, there is a need not only to monitor via experiment, insofar as it may be feasible, the following parameters for $\mathrm{Bi}-2212: n_{u 4}, s, n_{s}$ and $v_{c}$, but also to carry out similar studies for other SCs. Unfortunately, none of the conventionally employed critical state models sheds light on any of these parameters.

We conclude by noting that a detailed exposition of most of concepts of the BSE-based approach employed in this paper can be found in [24].

\section{Acknowledgements}

GPM thanks Dr. V.P.S. Awana for a valuable discussion of the conventional critical state models and the approach followed in this paper for determining the $j_{c}$ of an SC. It is gratifying to note that invoking the electronic structure of an SC in this context, as done here, seemed to be a refreshing new approach to him.

\section{Conflicts of Interest}

The authors declare no conflicts of interest regarding the publication of this paper.

\section{References}

[1] Tinkham, M. (1975) Introduction to Superconductivity. McGraw Hill, New York. 
[2] Ibach, H. and Lüth, H. (1996) Solid State Physics. Springer, Berlin. https://doi.org/10.1007/978-3-642-88199-2

[3] Bardeen, J. (1962) Critical Fields and Currents in Superconductors. Reviews of Modern Physics, 34, 667-681. https://doi.org/10.1103/RevModPhys.34.667

[4] Bean, C.P. (1964) Magnetization of High-Field Superconductors. Reviews of Modern Physics, 36, 31-39. https://doi.org/10.1103/RevModPhys.36.31

[5] Kim, Y.B., Hempstead, C.F. and Strand, A.R. (1963) Magnetization and Critical Supercurrents. Physical Review, 129, 528-535. https://doi.org/10.1103/PhysRev.129.528

[6] Kupriyanov, M.Y. and Lukichev, V.F. (1980) Temperature Dependence of PairBreaking Current in Superconductors. Soviet Journal of Low Temperature Physics, 6, 210.

[7] Lee, D. (2012) Iron-Based Superconductors: Nodal Rings. Nature Physics, 8, 364-365. https://doi.org/10.1038/nphys2301

[8] Zhang, Y., et al. (2012) Nodal Superconducting Gap-Structure in Ferropnictide Superconductor $\mathrm{Ba}_{2} \mathrm{Fe}_{2}\left(\mathrm{As}_{0.7} \mathrm{P}_{0.3}\right)_{2}$. Nature Physics, 8, 371-375. https://doi.org/10.1038/nphys2248

[9] Allan, M.P., et al. (2012) Anisotropic Energy Gaps of Iron-Based Superconductivity from Intraband Quasiparticle Interference in LiFeAs. Science, 336, 563-567. https://doi.org/10.1126/science.1218726

[10] Lin, X., et al. (2013) Fermi Surface of the Most Dilute Superconductor. Physical Review $X$, 3, Article ID: 021002. https://doi.org/10.1103/PhysRevX.3.021002

[11] Alexandrov, A.S. (2001) Nonadiabatic Polaronic Superconductivity in $\mathrm{MgB}_{2}$ and Cuprates. Physica C: Superconductivity, 363, 231-236. https://doi.org/10.1016/S0921-4534(01)01095-4

[12] Jarlborg, T. and Bianconi, A. (2013) Fermi Surface Reconstruction of Superoxygenated $\mathrm{La}_{2} \mathrm{CuO}_{4}$ Superconductors with Ordered Oxygen Interstitials. Physical Review $B, \mathbf{8 7}$, Article ID: 054514. https://doi.org/10.1103/PhysRevB.87.054514

[13] Malik, G.P. (2015) A Study of Heavy-Fermion Superconductors via BCS Equations Incorporating Chemical Potential. Journal of Modern Physics, 6, 1233-1242. https://doi.org/10.4236/jmp.2015.69128

[14] Malik, G.P. and Varma, V.S. (2015) A Study of Superconducting $\mathrm{La}_{2} \mathrm{CuO}_{4}$ via Generalized BCS Equations Incorporating Chemical Potential. World Journal of Condensed Matter Physics, 5, 148-159. https://doi.org/10.4236/wjcmp.2015.53017

[15] Malik, G.P. (2016) On the Role of Fermi Energy in Determining Properties of Superconductors: A Detailed Comparative Study of Two Elemental Superconductors $(\mathrm{Sn}$ and $\mathrm{Pb})$, a Non-Cuprate $\left(\mathrm{MgB}_{2}\right)$ and Three Cuprates (YBCO, Bi-2212 and Tl-2212). Journal of Superconductivity and Novel Magnetism, 29, 2755-2764. https://doi.org/10.1007/s10948-016-3637-5

[16] Malik, G.P. (2018) Correction to: On the Role of Fermi Energy in Determining Properties of Superconductors: A Detailed Comparative Study of Two Elemental Superconductors ( $\mathrm{Sn}$ and $\mathrm{Pb}$ ), a Non-Cuprate $\left(\mathrm{MgB}_{2}\right)$ and Three Cuprates (YBCO, Bi-2212 and Tl-2212). Journal of Superconductivity and Novel Magnetism, 31, 941-941. https://doi.org/10.1007/s10948-017-4520-8

[17] Malik, G.P. (2010) On Landau Quantization of Cooper Pairs in a Heat Bath. Physica $B, 405,3475-3481$. https://doi.org/10.1016/j.physb.2010.05.026

[18] Malik, G.P. (2013) On a New Equation for Critical Current Density in Terms of the BCS Interaction Parameter, Debye Temperature and the Fermi Energy of the Su- 
perconductor. World Journal of Condensed Matter Physics, 3, 103-110. https://doi.org/10.4236/wjcmp.2013.32017

[19] Poole, C.P. (2000) Handbook of Superconductivity. Academic Press, San Diego.

[20] Malik, G.P. and Varma, V.S. (2019) Generalized BCS Equations: A Review and a Detailed Study of the Superconducting Features of $\mathrm{Ba}_{2} \mathrm{Sr}_{2} \mathrm{CaCu}_{2} \mathrm{O}_{8}$. In: Superconductivity and Superfluidity, IntechOpen, London, $21 \mathrm{p}$. https://doi.org/10.5772/intechopen.84340

[21] Kumar, J., Sharma, D., Ahluwalia, P.K. and Awana, V.P.S. (2013) Enhanced Superconducting Performance of Melt Quenched $\mathrm{Bi}_{2} \mathrm{Sr}_{2} \mathrm{CaCu}_{2} \mathrm{O}_{8}$ (Bi-2212) Superconductor. Materials Chemistry and Physics, 139, 681-688. https://doi.org/10.1016/j.matchemphys.2013.02.016

[22] Malik, G.P. (2014) BCS-BEC Crossover without Appeal to Scattering Length Theory. International Journal of Modern Physics B, 8, Article ID: 1450054. https://doi.org/10.1142/S0217979214500544

[23] Malik, G.P. (2017) On the $\mathrm{S}^{ \pm}$-Wave Superconductivity in the Iron-Based Superconductors: A Perspective Based on a Detailed Study of $\mathrm{Ba}_{0.6} \mathrm{~K}_{0.4} \mathrm{Fe}_{2} \mathrm{As}_{2}$ via the Generalized Bardeen-Cooper-Schrieffer Equations Incorporating Fermi Energy. Open Journal of Composite Materials, 7, 130-145. https://doi.org/10.4236/ojcm.2017.73008

[24] Malik, G.P. (2016) Superconductivity: A New Approach Based on the Bethe-Salpeter Equation in the Mean-Field Approximation. Volume 21 in the Series on Directions in Condensed Matter Physics. World Scientific, Singapore.

https://doi.org/10.1142/9868 


\section{Appendix A}

In order to see that $\lambda_{m}$ defined in (8) in the natural system of units is dimensionless, we need the following conversion factors:

$$
\begin{aligned}
& 1 \mathrm{~g}=a_{1} e V c^{-2}, 1 \mathrm{~cm}=a_{2} e V^{-1}(\hbar c), e=b_{1}(\hbar c)^{1 / 2}, \\
& 1 \mathrm{~s}=a_{3} e V^{-1} \hbar, 1 \mathrm{G}=\mathrm{g}^{1 / 2} \cdot \mathrm{cm}^{-1 / 2} \cdot \mathrm{s}^{-1}=b_{2} e V^{2}(\hbar c)^{-3 / 2},
\end{aligned}
$$

where

$$
\begin{aligned}
& a_{1}=5.60958616 \times 10^{32}, a_{2}=5.06772886 \times 10^{4}, a_{3}=1.51926689 \times 10^{15} \\
& b_{1}=(137.03599895)^{-1 / 2}, b_{2}=a_{1}^{1 / 2} a_{2}^{-1 / 2} a_{3}^{-1}=6.92507774 \times 10^{-2} .
\end{aligned}
$$

Hence, we have

$$
\begin{gathered}
e=b_{1}(\hbar c)^{1 / 2}=b_{1}(\text { if } \hbar=c=1) \\
H_{c}(B C S, G)=b_{2}\left|H_{c}\right| e V^{2}(\hbar c)^{-3 / 2}=b_{2}\left|H_{c}\right| e V^{2}(\text { if } \hbar=c=1) \\
V_{B C S}=|V| e V c m^{3}=a_{2}^{3}|V| e V^{-2}(\hbar c)^{3}=a_{2}^{3}|V| e V^{-2}(\text { if } \hbar=c=1)
\end{gathered}
$$

It follows from the above equations that when $\hbar \neq 1$ and $c \neq 1, \mathrm{~V}_{\mathrm{BCS}}$ has to be divided by $(\hbar c)^{3}$ in order for it to play the required role of a propagator in the BSE-based approach and that, after inserting a factor of $\hbar c$ on the RHS of the first equation in the set of Equations (2), we obtain

$$
\lambda_{m}=\frac{a_{2}^{3} b_{1} b_{2}|H||V|}{16 \pi^{2}} \sqrt{\frac{2 m^{*} c^{2}}{E_{F}}},
$$

which is dimensionless, since both $|H|$ and $|V|$ are pure numbers.

We note that (A4) is needed only when one wishes to decouple the constituent $V$ of $\lambda_{m}$ after the latter has been determined corresponding to a given value of $H$. 